International Mathematical Forum, 2, 2007, no. 52, 2561 - 2570

\title{
Public Key Cryptography Based on Semimodules over Quotient Semirings
}

\author{
Reza Ebrahimi Atani \\ Department of Electrical Engineering \\ Iran University of Science and Technology \\ P.O. Box 16846, Tehran, Iran \\ rebrahimi@iust.ac.ir \\ Shahabaddin Ebrahimi Atani \\ Department of Mathematics \\ University of Guilan \\ P.O. Box 1914, Rasht, Iran \\ ebrahimi@guilan.ac.ir \\ Sattar Mirzakuchaki \\ Department of Electrical Engineering \\ Iran University of Science and Technology \\ P.O. Box 16846, Tehran, Iran \\ m-kuchaki@iust.ac.ir
}

\begin{abstract}
A generalization of the original Diffie-Hellman key exchange in $(Z / p Z)^{*}$ found a new depth when Miller [10] and Koblitz [7] suggested that such a protocol could be used with the group over an elliptic curve. Maze, Monico and Rosenthal extend such a generalization to the setting of a semigroup action on a finite set, more precisely, linear actions of abelian semirings on semimodules [8]. In this paper, we extend such a generalization to the linear actions of quotient semirings on semimodules. In fact, we show how the action of a quotient semirings on a semimodule gives rise to a generalized Diffie-Hellman and ElGamal protocol. This leads naturally to a cryptographic protocol whose difficulty is based on the hardness of a particular control problem, namely the problem of steering the state of some dynamical system from an intial vector to some final location.
\end{abstract}


Keywords: Public key cryptography, Deffie-Helman protocol, One way trapdoor functions, Semigroup actions, Quotient semirings

\section{Introduction}

The Diffie-Hellman key exchange and the ElGamal oneway trapdoor function are the basic ingredients of public key cryptography. Both these protocols are based on the hardness of the discrete logarithm problem in a finite semiring. The discrete logarithm problem, commonly abbreviated $D L P$, is a recurrent tool in public-key cryptography. The problem takes place in any group $G$, but we shall always assume the group is finite and commutative.

Protocol 1.1 [The Discrete Logarithm Problem - DLP] Let $G$ be a finite commutative group. Given two group elements $a$ (the base) and $b$ such that that $b \in\langle a\rangle$, find $0 \leq n \leq \operatorname{ord}(a)$ such that $a^{n}=b$. We denote such an $n$ by $\log _{a} b$.

For cryptographic purpose, we will always assume that the group $G$ is presented in such a way that multiplication is computationally easy. Note that this requirement makes exponentiation feasible as well using well-known methods of type square-and multiply (see [9] or [11]).

The difficulty of the $D L P$ strongly depends on the type of group that is used: It goes from easy to non-feasible. For instance the $D L P$ in the additive group of any finite field $F_{q}$ is trivial since division can be performed in polynomial time. However, the $D L P$ in the multiplicative group $F_{q}^{*}$ is a difficult problem as well as the $D L P$ in the group $E\left(F_{q}\right)$ of an elliptic curve defined over a finite field. In fact the latter is much more difficult than the former and intuition tells us that the less structure the group has, the more difficult that $D L P$ will be. Protocols where the discrete logarithm problem plays a significant role are the Diffie-Hellman key agreement [2], the ElGamal public key cryptosystem [3], the digital signature algorithm $(D S A)$ and ElGamal's signature scheme [9]. This is one of the reasons why we have developed the ideas of the this paper. In the sequel we outline two of these protocols and we refer the interested reader to [9] for further details.

The Diffie-Hellman protocol [2] allows two parties, say Alice and Bob, to exchange key over some insecure channel. In order to achieve this goal Alice and bob agree on a group $G$ and a common base $g \in G$. Alice chooses a random positive integer $a$ and Bob chooses a random positive integer $b$. Alice transmits to Bob $g^{a}$ and Bob transmits to Alice $g^{b}$. Their common secret key is $k=g^{a b}$. 
The ElGamal public key cryptosystem [3] works in the following way: Alice chooses positive integer $n$ and $h, g \in G$, where $h=g^{n}$. the private key of Alice consists of $(g, h, n)$, the public key consists of $(g, h)$. Bob chooses a random positive integer $r$ and with this he applies the encryption function $v: G \rightarrow G \times G$ (sending $m$ to $\left.\left(c_{1}, c_{2}\right)=\left(g^{r}, m h^{r}\right)\right)$. Alice, who knows $n=\log _{g} h$ readily computes $m$ from the cipher text $\left(c_{1}, c_{2}\right): m=c_{2}\left(c_{1}^{n}\right)^{-1}$. In order for the protocol to work it is required that multiplication and inversion inside the group $G$ can be efficiently done and it should be computationally infeasible to compute a discrete logarithm with base $g \in G$.

In [8], Maze, Monico and Rosenthal have shown how the discrete logarithm problem over a group can be seen as a special instance of an action by a semigroup. In fact, they have shown every semigroup action by an abelian semigroup gives rise the a Diffie-Hellman key exchange. With an additional assumption it is also possible to extended the ElGamal protocol. Let us explain them in detail. Assume that $S$ is a finite set and let $G$ be a semigroup. Consider an action of $G$ on $S: G \times S \rightarrow S$ (sending $(g, s)$ to $g s$ ). By the definition of a group action we require that $(g h) s=g(h s)$ for all $g, h \in G$ and $s \in S$. We also assume throughout that arithmetic in $G$ and computation of the $G$-action can be done in polynomial time. If the semigroup $G$ is commutative then every $G$-action gives rise to a generalized Diffie-Hellman Key Exchange [8]:

Protocol 1.2 (Extended Diffie-Hellman Key Exchange) Let $S$ be a finite set, $G$ a commutative semigroup and an action of $G$ on $S$ as defined above. The Extended Diffie-Hellman Key Exchange is the following protocol [8]:

1) Alice and Bob agree on an element $s \in S$.

2) Alice chooses $a \in G$ and computes as. Alice's secret key is $a$, her public key is as.

3) Bob chooses $b \in G$ and computes $b s$. Bob's secret key is $b$, his public key is $b s$.

4)Their common secret key is then $a(b s)=(a b) s=(b a) s=b(a s)$.

Protocol 1.3 (Extended ElGamal Public Key System) Let $S$ be a group with respect to some operation $o, G$ an abelian semigroup and an action of $G$ on $S$ as defined above. The Extended ElGamal Public Key System is the following protocol [8]:

1) Alice's public key is $(s, a s)$.

2) Bob chooses a random element $b \in G$ and encrypts a message $m$ using the encryption function

$$
(m, b) \longmapsto(b s,(b(a s)) \mathrm{o} m)=\left(c_{1}, c_{2}\right)
$$


3) Alice can decrypt the message using $m=(b(a s))^{-1}$ o $c_{2}=\left(a c_{1}\right)^{-1}$ o $c_{2}$.

In [8] Maze, Monico and Rosenthal show how to build semigroup actions from actions by semirings on semimodules. In this paper we show how to build semigroup actions from actions by quotient semirings on semimodules.

\section{Quotient semirings acting on semimodules}

A set $R$ together with two associative binary operations called addition and multiplication (denoted by + and ., respectively) will be called a semiring provided 1) addition is a commutative operation and that the multiplication is distributive with respect to the addition both from the left and from the right; 2) there exists $0 \in R$ such that $r+0=r$ and $r .0=0 . r=0$ for all $r \in R$. A subset $I$ of a semiring $R$ will be called an ideal if $a, b \in I$ and $r \in R$ implies $a+b \in I$ and $r a, a r \in I$. A subtractive ideal (= $k$-ideal) $K$ is an ideal such that if $x, x+y \in I$ then $y \in K$. A (left) semimodule $M$ over a semiring $R$ is a commutative additive semigroup which has a zero element, together a mapping from

$$
R \times M \rightarrow M
$$

sending $(r, m)$ to $r m$ such that $(r+s) m=r m+s m, r(m+p)=r m+r p)$, $r(s m)=(r s) m$ and $0 m=r 0_{M}=0_{M}$ for all $m, p \in M$ and $r, s \in R$.

An ideal $I$ of a semiring $R$ is called a partitioning ideal ( $=Q$-ideal) if there exists a non-empty subset $Q$ of $R$ such that

(1) $R=\cup\{q+I: q \in Q\}$;

(2)If $q_{1}, q_{2} \in Q$ then $\left(q_{1}+I\right) \cap\left(q_{2}+I\right) \neq \emptyset$ if and only if $q_{1}=q_{2}$.

Let $I$ be a $Q$-ideal of a semiring $R$ and let $R / I=\{q+I: q \in Q\}$. Then $R / I$ forms a semiring under the binary operations $\oplus$ and $\odot$ defined as follows: $\left(q_{1}+I\right) \oplus\left(q_{2}+I\right)=q_{3} I$ where $q_{3} \in Q$ is the unique element such that $q_{1}+q_{2}+I \subseteq q_{3}+I$ and $\left(q_{1}+I\right) \odot\left(q_{2}+I\right)=q_{4}+I$ where $q_{4} \in Q$ is the unique element such that $q_{1} q_{2}+I \subseteq q_{4}+I$. This semiring $R / I$ is called the quotient semiring of $R$ by $I$. By definition of $Q$-ideal, there exists a unique $q_{0} \in Q$ such that $0+I \subseteq q_{0}+I$. Then $q_{0}+I$ is a zero element of $R / I[6,4]$. it is well-known that if $R$ is a semiring, then $\operatorname{Mat}(R)$, the set of $n \times n$ matrices with entries in $R$ is a semiring.

Let $M$ be a finite semimodule over a semiring $R$, and let $I$ be a $Q$-ideal of $R$. Now let $r \in R$ and suppose that $q_{1}+I, q_{2}+I \in R / I$ are such that $q_{1}+I=q_{2}+I$ in $R / I$. Then $q_{1}=q_{2}$, we must have $q_{1} m=q_{2} m$ for every $m \in M$. Hence we can unambiguously define a mapping $R / I \times M$ into $M$ (sending $(q+I, m)$ to 
$q m$ ) and it is routine to check that this turns the commutative semigroup $M$ into an $R / I$-semimodule.

Convention. The remaining of this paper we will assume unless otherwise stated, if $I$ is an $Q$-ideal of $R$, then $Q$ is closed under addition and multiplication of $R$.

Let $\operatorname{Mat}(R / I)$ be the set of all $n \times n$ matrices with entries in $R / I$. The semiring structure on $R / I$ induces a semiring structure on $\operatorname{Mat}(R / I)$. Moreover the semimodule structure on $M$ lifts to a semimodule structure on $M^{n}$ via the matrix multiplication:

$$
\operatorname{Mat}(R / I) \times M^{n} \rightarrow M^{n}
$$

sending $(A, x)$ to $A x)$ where $x$ is a $n \times 1$ matrix with entries $m_{11}, \ldots, m_{n 1}$ and $A=\left(q_{i j}+I\right)_{n \times n}$ with $q_{i j} \in Q$ for every $i, j$. One readily verifies that

$$
\operatorname{Mat}(R / I) \times M^{n} \rightarrow M^{n}
$$

is an action by a semi-group, indeed one readily computes that $A(B x)=$ $(A B) x$. Let us explain this equality in more detail. For simplicity, assume that $n=2$ and let $A=\left(a_{i j}+I\right)_{2 \times 2}, B=\left(b_{i j}+I\right)_{2 \times 2}$ and $x=\left(m_{i 1}\right)_{2 \times 1}$. Let $A(B x)=\left(c_{i j}\right)_{2 \times 1}$. Then we must have

$$
\begin{aligned}
& a_{11} b_{11} m_{11}+a_{11} b_{12} m_{21}+a_{12} b_{21} m_{11}+a_{12} b_{22} m_{21}=c_{11} \\
& a_{21} b_{11} m_{11}+a_{21} b_{12} m_{21}+a_{22} b_{21} m_{11}+a_{22} b_{22} m_{21}=c_{21}
\end{aligned}
$$

Let $A B=\left(e_{i j}+I\right)_{2 \times 2}$. Then we must have

$$
\begin{aligned}
& \left(a_{11}+I\right) \odot\left(b_{11}+I\right) \oplus\left(a_{12}+I\right) \odot\left(b_{21}+l\right)=e_{11}+l \\
& \left(a_{11}+I\right) \odot\left(b_{12}+I\right) \oplus\left(a_{12}+I\right) \odot\left(b_{22}+l\right)=e_{12}+l \\
& \left(a_{21}+I\right) \odot\left(b_{11}+I\right) \oplus\left(a_{22}+I\right) \odot\left(b_{21}+l\right)=e_{21}+l \\
& \left(a_{21}+I\right) \odot\left(b_{12}+I\right) \oplus\left(a_{22}+I\right) \odot\left(b_{22}+l\right)=e_{22}+l
\end{aligned}
$$

It then follows from (3) that there are unique elements $d_{11}, d_{12}$ of $Q$ such that

$$
\left(d_{11}+I\right) \oplus\left(d_{12}+I\right)=e_{11}+l
$$


where $a_{11} b_{11}+I \subseteq d_{11}+I, a_{12} b_{21}+I \subseteq d_{12}+I$ and $d_{11}+d_{12}+I \subseteq e_{11}+I$; hence

$$
a_{11} b_{11}+a_{12} b_{21}=d_{11}+d_{12}=e_{11}
$$

since $I$ is a $Q$-ideal of $R$. Similarly, the relations (4), (5) and (6) gives

$$
a_{11} b_{12}+a_{12} b_{22}=e_{12}, a_{21} b_{11}+a_{22} b_{21}=e_{21}, a_{21} b_{12}+a_{22} b_{22}=e_{22} .
$$

Let $(A B) x=\left(e_{i j}+I\right)_{2 \times 2}\left(m_{i 1}\right)_{2 \times 1}=\left(f_{i 1}\right)_{2 \times 1}$. Then we must have

$$
e_{11} m_{11}+e_{12} m_{21}=f_{11}, e_{21} m_{11}+e_{22} m_{21}=f_{21}
$$

Now the relation (1), (2), (8), (9) and (10) gives

$$
\begin{aligned}
& f_{11}=a_{11} b_{11} m_{11}+a_{11} b_{12} m_{21}+a_{12} b_{21} m_{11}+a_{12} b_{22} m_{21}=c_{11} \\
& f_{21}=a_{21} b_{11} m_{11}+a_{21} b_{12} m_{21}+a_{22} b_{21} m_{11}+a_{22} b_{22} m_{21}=c_{21}
\end{aligned}
$$

Thus $(A B) x=A(B x)$.

Remark Assume that $I$ is an $Q$-ideal of $R$ such that $a b=b a$ for all $a, b \in Q$ and let $q_{I}, q^{\prime}+I \in R / I$. Then there are unique elements $c, c^{\prime} \in Q$ with $q q^{\prime}+I \subseteq c+I$ and $q^{\prime} q+I \subseteq c^{\prime}+I$, so $(q+I) \odot\left(q^{\prime}+I\right)=\left(q^{\prime}+I\right) \odot(q+I)$ since $q q^{\prime}=q^{\prime} q$; hence $R / I$ is a commutative ring.

Assume that $I$ is an $Q$-ideal of $R$ such that $q q^{\prime}=q^{\prime} q$ for all $q, q^{\prime} \in Q$ and set $\bar{R}=R / I=\{q+I: q \in Q\}=\{\bar{q}: q \in Q\}$. Let $\bar{R}[t]$ be the polynomial semiring in the in determinant $t$, and let $A \in \operatorname{Mat}(\bar{R})$ be a fixed matrix. If

$$
\bar{p}(t)=\bar{q}_{0}+\bar{q}_{1} t+\ldots+\bar{q}_{k} t^{k} \in \bar{R}[t]
$$

then we define in the usual way $\bar{p}(A)=\bar{q}_{0} I_{n}+\bar{q}_{1} A+\ldots+\bar{q}_{k} A^{k}$, where $\bar{q}_{0} I_{n}$ is the $n \times n$ diagonal matrix with entry $\bar{q}_{0}$ in each diagonal element. Consider the semigroup

$$
\bar{G}=\bar{R}[A]=\{\bar{p}(A): \bar{p}(t) \in \bar{R}[t]\}
$$

It is easy to see that $\bar{G}$ has the structure of an abelian semigroup.

Protocol 2.1 then simply requires that Alice and Bob agree on an $Q$-ideal $I$ of a semiring $R$, an element $x \in \in M^{n}$ and a matrix $A \in \operatorname{Mat}(\bar{R})$. Alice chooses secretly $\bar{p}(t) \in \bar{R}[t]$ and computes $\bar{p}(A) x$ and sends the result to Bob. Bob chooses secretly $\bar{q}(t) \in \bar{R}[t]$ and computes $\bar{q}(A) x$ and sends the result to Alice. As a common secret key serves $k=\bar{p}(A) \bar{q}(A) x$ since $\bar{p}(A)$ and $\bar{q}(A)$ commute. 
System Theoretic Interpretation. It is possible to give the key exchange a systems theoretic interpretation. For this note that in order to choose $\bar{p}(A) \in \bar{R}[A]$ Alice has to choose $\bar{q}_{0}=q_{0}+I, \ldots, \bar{q}_{k}=q_{k}+I \in \bar{R}=R / I$ and with this she can compute

$$
\bar{p}(A) x=\left(\bar{q}_{0}+\bar{q}_{1} A+\ldots+\bar{q}_{k} A^{k}\right) x=\bar{q}_{0} x+\bar{q}_{1} A x+\ldots+\bar{q}_{k} A^{k} x
$$

Consider now the linear time invariant system:

$$
y_{t+1}=A y_{t}+\bar{u}_{t} x
$$

where $x, y_{t} \in M^{n}$ and $\bar{u}_{t} \in \bar{R}$.

Suppose further that $y_{0}=0_{M}$. If Alice chooses the input sequence $\bar{u}_{0}=\bar{q}_{k}$, $\bar{u}_{1}=\bar{q}_{k-1}, \ldots, \bar{u}_{k}=\bar{q}_{0}$ then $\bar{y}_{k+1}$, the state vector at time $k+1$ is exactly $\bar{p}(A) x$ the public vector to be computed by Alice. Once Alice receives from Bob his public key $\bar{f}(A) x$, then she defines $b=\bar{f}(A) x$ and by choosing her input sequences $\bar{u}_{0}, \ldots, \bar{u}_{k}$ in the system $y_{t+1}=A y_{t}+\bar{u}_{t} b$. Then she will be able to compute the common secret key $\bar{p}(A) \bar{f}(A) x$.

adversary who want to find an element $\bar{g}(t) \in \bar{R}[t]$ such that $\bar{g}(A) x=$ $\bar{p}(A) x)$ faces the task of finding a control sequence $\bar{u}_{0}, \ldots, \bar{u}_{k}$ which steers the initial state $y_{0}$ in to the state $\bar{p}(A) x$. This problem is in general very hard, but it contains some of the hardest known discrete logarithm problem as a special case. For example, when $R / I=M=F$, a finite field then the problem is however simply solved by [8, Theorem 3.1].

\section{Matrix quotient semirings acting on semi- modules}

Assume that $R$ is a semiring and let $\operatorname{Mat}(R)$ be the set of $n \times n$ matrices with entries in $R$. Our starting point in this section is the following theorem:

Theorem 3.1 Let I be a $Q$-ideal of a semiring $R$. Then $\operatorname{Mat}_{n}(I)$ is a $\operatorname{Mat}(Q)$ ideal of $\operatorname{Mat}(R)$. In particular, $\operatorname{Mat}(R) / \operatorname{Mat}(I)=\{C+\operatorname{Mat}(I): C \in \operatorname{Mat}(Q)\}$ is a semiring.

Proof. It is easy to see that $\operatorname{Mat}(I)$ is an ideal of $\operatorname{Mat}(R)$. Since the inclusion $\cup\{q+\operatorname{Mat}(I): q \in \operatorname{Mat}(Q)\} \subseteq \operatorname{Mat}(R)$ is trivial, we will prove the reverse inclusion. Suppose that $A=\left(a_{i j}\right)_{n \times n} \in \operatorname{Mat}(R)$. Then there are elements $q_{i j} \in Q$ and $c_{i j} \in I$ such that $a_{i j}=q_{i j}+c_{i j}$ for all $i, j$ since $I$ is a $Q$-ideal of $R$. Set $B=\left(q_{i j}\right)_{n \times n}$ and $C=\left(c_{i j}\right)_{n \times n}$. Then $A=B+C \in \operatorname{Mat}(Q)+\operatorname{Mat}(I)$, and so we have equality. Suppose that $\left.\left(E+\operatorname{Mat}_{(} I\right)\right) \cap(F+\operatorname{Mat}(I)) \neq \emptyset$ where 
$E=\left(e_{i, j}\right)_{n \times n}, F=\left(f_{i, j}\right)_{n \times n} \in \operatorname{Mat}_{n \times n}(Q)$; we show that $E=F$. There exist $H=\left(h_{i j}\right)_{n \times n}, K=\left(k_{i j}\right)_{n \times n} \in \operatorname{Mat}_{n \times n}(I)$ such that $E+H=F+K$, so for all $i, j,\left(e_{i j}+I\right) \cap\left(f_{i j}+I\right) \neq \emptyset$; hence $E=F$ since $I$ is a $Q$-ideal, as needed.

Let $M$ be a finite semimodule over a semiring $R$. The semimodule structure on $M$ lifts to a semimodule structure on $M^{n}$ via the matrix multiplication:

$$
\operatorname{Mat}(R) \times M^{n} \rightarrow M^{n}
$$

sending $(A, x)$ to $A x[8]$.

If $I$ is an $Q$-ideal of $R$, then Theorem 3.1 gives $\operatorname{Mat}(R) / \operatorname{Mat}(I)$ is a semiring. Moreover, if $I$ is closed under addition and multiplication of $R$, then it is easy to see that $\operatorname{Mat}(I)$ is closed under addition and multiplication of $\operatorname{Mat}(R)$. Now the matrix multiplication:

$$
\operatorname{Mat}(R) / \operatorname{Mat}(I) \times M^{n} \rightarrow M^{n}
$$

sending $(A+\operatorname{Mat}(I), x)$ to $A x$ is a semimodule structure on $M^{n}$ where $A \in$ $\operatorname{Mat}(Q)$. One readily verifies that

$$
\operatorname{Mat}(R) / \operatorname{Mat}(I) \times M^{n} \rightarrow M^{n}
$$

is an action by a semi-group, indeed one readily computes that $A(B x)=$ $(A B) x$. Let us explain this equality in more detail. Let $A=\left(a_{i j}\right)_{n \times n}+\operatorname{Mat}(I)$, $B=\left(b_{i j}\right)_{n \times n}+\operatorname{Mat}(I)$ and $x=\left(m_{i 1}\right)_{n \times 1}$ where $a_{I j}, b_{i j} \in Q$. Then we must have

$$
A(B x)=\left(a_{i j}\right)_{n \times n}\left(b_{i j}\right)_{n \times n}\left(m_{i 1}\right)_{n \times 1}
$$

Let $A B=\left(e_{i j}\right)_{n \times n}+\operatorname{Mat}(I)$. Then we must have

$\left(a_{i j}\right)_{n \times n}\left(b_{i j}\right)_{n \times n}+\operatorname{Mat}(I) \subseteq\left(e_{i j}\right)_{n \times n}+\operatorname{Mat}(I)$, so we get $\left(a_{i j}\right)_{n \times n}\left(b_{i j}\right)_{n \times n}=$ $\left(e_{i j}\right)_{n \times n}$ since $\operatorname{Mat}(I)$ is a $\operatorname{Mat}(Q)$ - ideal of $\operatorname{Mat}(R)$. It follows that

$$
(A B) x=\left(a_{i j}\right)_{n \times n}\left(b_{i j}\right)_{n \times n}\left(m_{i 1}\right)_{n \times 1}
$$

Now the assertion follows from (13) and (14).

Assume that $S=\operatorname{Mat}(R) / \operatorname{Mat}(I)$ and let $S[t]$ be the polynomial semiring in the in determinant $t$ and let $A=\left(a_{i j}\right)_{n \times n}+\operatorname{Mat}(I) \in S$ be a fixed member. Let $C \subseteq S$ be the center of $S$. If $p(t)=r_{0}+r_{1} t+\ldots, r_{k} t^{k} \in C[t]$, then we define in the usual way $p(A)=r_{0} I_{n}+r_{1} A+\ldots, r_{k} A^{k}$. Then $C[A]=\{p(A): p(t) \in C[t]\}$ has the structure of an abelian semigroup. Alice and Bob agree on an $R$ semimodule $M$, an element $x \in M^{n}$ and an element $A$ of $S$. Alice chooses 
secretly $p(t) \in C[t]$ and computes $p(A) x$ and sends the result to Bob. Bob chooses $q(t) \in C[t]$ and computes $q(A) x$ and sends the result to Alice. As a common key serves $k=p(A) q(A) x$. It should be difficult to find $g(t) \in C[t]$ such that $g(A) x=p(A) x$.

\section{Conclusion}

At present, we lack a convincing example of a system based on the previous sections. All of the examples has presented in [8] to be either insecure or already well-known. The insecure examples have arisen by generating random finite semiring for base-objects. Of course, there are some strong results in [8] on simple semimodules over commutative semirings, but more work is needed to determine if there exists such objects that will suit our needs. In this paper we showed how the discrete logarithm problem over a finite group can viewed as an instance of an action by a semigroup. In fact, we show how the action of a quotient semiring on a semimodule gives rise to a generalized Diffe-Hellman and ElGamal protocol. It remains to find concrete instances of such actions that have high (believed) security relative to their key size.

\section{References}

[1] P. J. Allen, A fundamental theorem of homeomorphisms for simirings, Proc. Amer. Math. Soc., 21 (1969), 412-416.

[2] W. Diffie and M. E. Hellmann, New directions in cryptography, IEEE Trans. Inform. Theory, IT-22 (6) (1976), 644-654.

[3] T. ElGamal, A public key cryptosystem and a signature scheme based on discrete logarithm, IEEE Trans. Inform. Theory, 31 (4) (1985), 469-472.

[4] Shahabaddin Ebrahimi Atani, The ideal theory in quotients of commutative semirings, Glasnik Matematicki, to appear (2007).

[5] Shahabaddin Ebrahimi Atani and Reza Ebrahimi Atani, Prime subsemimodules of semimodules, submitted.

[6] V. Gupta and J. N. Chaudhari, Some remarks on Right $\pi$-regular semirings, Sarajevo J. of Math., 14 (2006), 3-9.

[7] N. Koblitz, Elliptic curve cryptosystem, Math. Comp., 48(177) (1986), 203-209. 
[8] G. Maze, C. Monico and J. Rosenthal, Public Key Cryptography On Semigroup Actions, arXiv:cs. CR/0501017v2 28 Jan 2005.

[9] A. J. Meenezes and P. C. van Oorschot, and S. A. Vanstone, Handbook of Applied Cryptography, CRC Press Series on Discrete Mathematics and its Applications 1997. .

[10] V. S. Miller, Use of elliptic curves in cryptography, In Advances in cryptology - CRYPTO 85 (Santa Barbara, Calif., 1985), Pages 417426, Springer, Berlin, 1986.

[11] Song Y. Yan, Number theory for computing, Springer-Verlag, Berlin, 2000.

Received: May 7, 2007 